\title{
Performances and main results of the KM3NeT prototypes
}

\author{
Simone Biagi ${ }^{* \dagger}$ \\ INFN, Laboratori Nazionali del Sud, Catania, Italy \\ E-mail: biagi@bo.infn.it
}

\section{Robert Bormuth}

Nikhef, Amsterdam, The Netherlands

E-mail: rbormuth@nikhef.nl

\section{Alexandre Creusot}

APC, Université Paris Diderot, CNRS/IN2P3, CEA/IRFU, Observatoire de Paris, France

E-mail: creusoteapc.in2p3.fr

The KM3NeT collaboration aims to build a km3-scale neutrino telescope in the Mediterranean Sea. The first phase of construction comprises the deep-sea and onshore infrastructures at the KM3NeT-It (100 km offshore Capo Passero, Italy) and KM3NeT-Fr (40 km offshore Toulon, France) sites and the installation of $31+7$ detection units. For the next step (KM3NeT 2.0) completion of two detectors are planned as extension of the detectors realized during the first phase of construction: ARCA for high energies $(\mathrm{E}>\mathrm{TeV})$ in Italy and ORCA for low energies $(\mathrm{GeV}$ range) in France.

A prototype digital optical module made of 31" PMTs was deployed in April 2013 inside the ANTARES neutrino telescope. This prototype, attached to an ANTARES string, was operated for one year and a half. It validated the multi-PMT technology and demonstrated the capability to identify muons with a single optical module searching for local time coincidences between PMTs inside the optical module.

A prototype detection unit made of three optical modules was installed at the KM3NeT-It site. It was deployed in May 2014; it is active and taking data. More than 700 hours of data have been recorded and analyzed. The experience achieved with this prototype detection unit validates the submarine deployment procedures, the mechanics and the electronic of the apparatus, the data taking and analysis procedures. Through the study of ${ }^{40} \mathrm{~K}$ decay in sea water and dedicated data taking periods with flashing LED nanobeacons, it is possible to calibrate in time the detector with nanosecond stability. A dedicated algorithm has been developed to select atmospheric muons and reconstruct their zenith angle with a resolution of about 8 degrees. An excellent agreement is found when comparing the detected signal from muons with Monte Carlo simulations.

The performance and results of the two prototypes will be presented.

The 34th International Cosmic Ray Conference,

30 July- 6 August, 2015

The Hague, The Netherlands

\footnotetext{
* Speaker.

${ }^{\dagger}$ On behalf of the KM3NeT Collaboration.
} 


\section{Introduction}

The KM3NeT collaboration has started the construction of a multi-site underwater detector to measure and identify neutrino interactions [1]. The first phase of construction is foreseen to install two 3-dimensional arrays of photomultipliers in two different marine sites close to Capo Passero, Italy, and Toulon, France. The two arrays of photomultipliers will have peculiar geometries optimized to detect neutrinos of different energies and perform distinct kinds of physics: the detection of high energy ( $>\mathrm{TeV}$ ) cosmic neutrinos in Italy (ARCA, Astroparticle Research with Cosmics in the Abyss), and low energy $(\sim \mathrm{GeV})$ neutrinos in France to determine the mass hierarchy of neutrinos (ORCA, Oscillations Research with Cosmics in the Abyss).

In both cases, the basic element of the apparatus is the Digital Optical Module (DOM), a 17" glass sphere which contains 31 photomultipliers of 3" diameter and the front-end electronics (Fig. 1a); the total photocathode surface is $1260 \mathrm{~cm}^{2}[2,3]$. The DOMs are arranged in vertical lines kept taut by a system of buoys and anchored on the sea bed. The detection unit for ARCA will be composed of 18 DOMs vertically spaced by $36 \mathrm{~m}$; the full ARCA apparatus comprises more than 200 detection units. The ORCA detector foresees about 100 detection units with DOMs spaced by $6 \mathrm{~m}$.

In these proceedings the main results from two prototype projects are presented: the prototype DOM (formerly known as the Pre-Production Model DOM, PPM-DOM) and the prototype detection unit (Pre-Production Model Detection Unit, PPM-DU). The latter is a string composed by only three DOMs with a vertical spacing of $36 \mathrm{~m}$.

\section{Prototype Digital Optical Module}

The prototype DOM [4] was deployed close to the Toulon coast, France $\left(42^{\circ} 50^{\prime} \mathrm{N}, 6^{\circ} 10^{\prime} \mathrm{E}\right)$ attached to the instrumented line of the ANTARES detector [5]. The PPM-DOM was operated at a depth of $2375 \mathrm{~m}$ since its deployment the $16^{\text {th }}$ of April 2013 up to the middle of November 2014 for the disconnection of the ANTARES line. The DOM was correctly working during the full period of operation.

The PPM-DOM houses 31 ETEL D783FL PMTs [6]; each PMT is surrounded by a cone reflector ring that increases its photo-detection area [7]. The PMTs are arranged inside the glass sphere in 5 rings with zenith angles of $56^{\circ}, 72^{\circ}, 107^{\circ}, 123^{\circ}$, and $148^{\circ}$, each ring containing 6 PMTs spaced at $60^{\circ}$ in azimuth. Subsequent rings have the PMTs shifted of $30^{\circ}$ in azimuth. The last PMTs is looking downward at the zenith angle of $180^{\circ}$.

The signal from the PMTs is digitized and timestamped by a multi-channel TDC (Time to Digital Converter) designed in the firmware of an FPGA that controls and operates all the electronics available inside the DOM [3]. The signals from all 31 PMTs is arranged in timeslices of $\sim 134 \mathrm{~ms}$. A threshold for the digitization of the signal is set at the level of 0.3 photoelectron. The TDC digitized the arrival time of the pulse and measures its Time over Threshold (ToT). A measurement of time and energy is simultaneously provided, considering that the width of the pulse is almost proportional to the number of photoelectrons collected. Finally data are transported onshore through the ANTARES optical link where they are stored on disk. Full raw data are recorded; neither triggering nor filtering algorithm is applied in this prototype. 


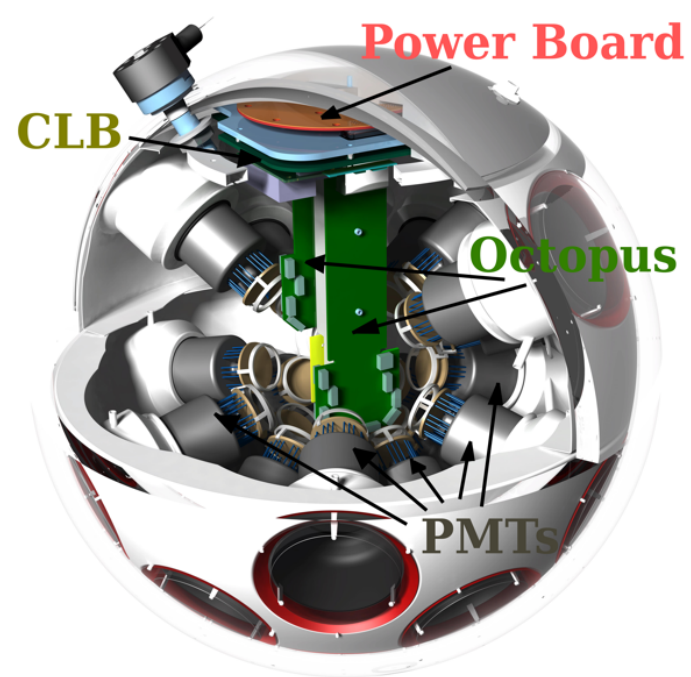

(a)

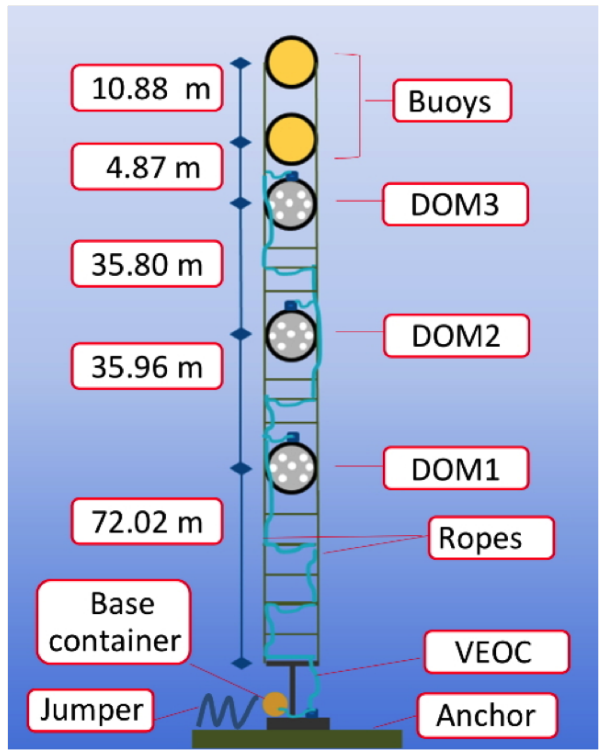

(b)

Figure 1: (a) A drawing of the Digital Optical Module. The internal structure is visible: the electronic boards to operate the PMTs and communicate on-shore (top), two "Octopus boards" to power the PMTs and connect them with the Central Logic Board (CLB), some of the 31 PMTs and the glass sphere [2,3]. (b) Graphical representation of the PPM-DU installed in the deep water (vertical and horizontal dimensions not in scale). The 3 DOMs are vertically spaced by $36 \mathrm{~m}$; two empty glass spheres are the buoys of the system; the electro-optical cable, represented with the cyan line, connects the DOMs with the base container and the latter with the onshore station.

An in situ time calibration of the 31 PMTs is done searching for local coincidences between couples of PMTs. The signal used for the intra-DOM time calibration is related to the decay in the sea water of the radioactive isotope ${ }^{40} \mathrm{~K}$. $\beta$-electrons and $\gamma$-rays produced in the ${ }^{40} \mathrm{~K}$ decay induce Cherenkov radiation; this process constitutes an isotropic source of $\sim 100$ detectable photons per ${ }^{40} \mathrm{~K}$ decay and it is the main cause of the baseline single rates measured in the PMTs. The DOM can detect multiple photons for a single ${ }^{40} \mathrm{~K}$ decay. The distributions of time differences between adjacent PMTs show a clear peak around the time offset value that indicates the detection of two photons from the same ${ }^{40} \mathrm{~K}$ decay [4]. This peak is less prominent when the angular separation between PMTs increases and eventually disappears when the PMTs are positioned back to back.

Using only one DOM, it is possible to select a sample of atmospheric muons almost background free, even though the efficiency of such selection is low. Data have been compared with a full Monte Carlo simulation that takes into account the relevant physics processes and the detector response. In particular, the simulation of the atmospheric muons was performed using a parameterization of the measured muon flux and multiplicity for the $2375 \mathrm{~m}$ depth [8]. The expected photon rate due to the ${ }^{40} \mathrm{~K}$ decay has been taken into account [9]. In the following, the coincidence level is defined as the number of PMTs that detect a photon in a coincidence time window of $20 \mathrm{~ns}$.

Fig. 2a shows the event rate as a function of the coincidence level. Below a coincidence level of six, the measured event rate is essentially due to the ${ }^{40} \mathrm{~K}$ decay and is in good agreement 


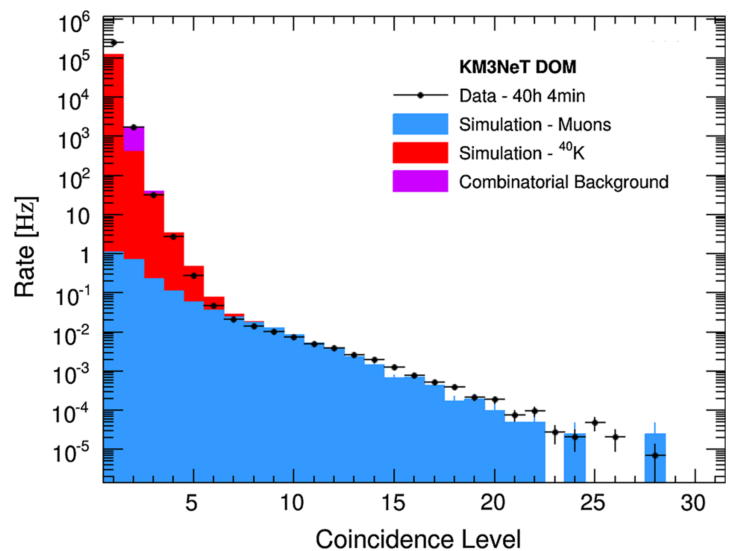

(a)

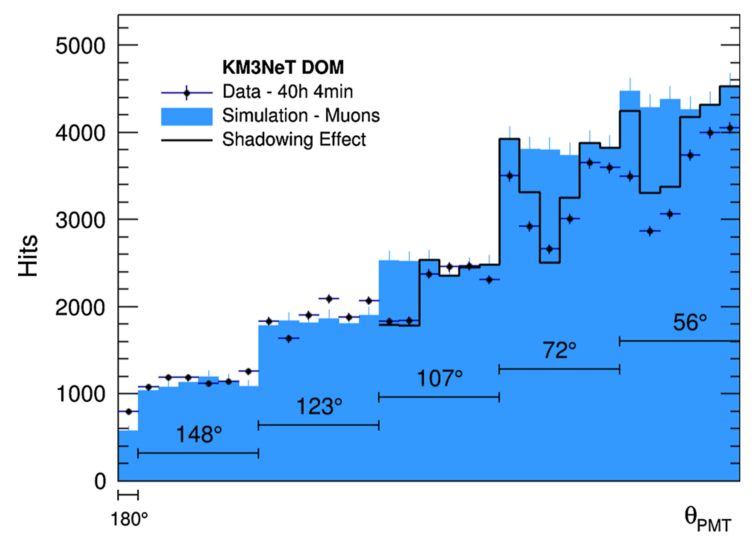

(b)

Figure 2: (a) Rate of recorded events as a function of the number of PMTs with signal in coincidence. Black dots are data, colored histograms represent simulations (muons in blue, ${ }^{40} \mathrm{~K}$ in red and combinatorial background in purple). (b) Number of hits as a function of the zenith position of the PMTs for events with more than seven coincidences. Data are the black dots, the simulation of atmospheric muons is represented by the blue histogram (the black histogram) without (with) the effect of the shadowing from the ANTARES electronics cylinder.

with the event rate given by the simulations. The singles rate has an additional contribution from bioluminescence (not simulated here). The muon simulation shows a good agreement with the data in the high coincidence level region. It is worthy to notice that the signal from atmospheric muons start to dominate above the coincidence level of seven. To sum up, it is possible to select a sample of pure muons with a single DOM using coincidences of eight PMTs.

The potential directionality of the multi-PMT optical module is evident in Fig. 2b. Here a cut was applied at a coincidence level larger than seven. In the figure the number of hits detected by each PMT is shown as a function of the position in terms of zenith angle, corresponding to the rings of PMTs in the DOM. The first PMT is the one looking downward $\left(\theta=180^{\circ}\right)$. Since atmospheric muons come from above, the number of events increases when the zenith angle of the PMTs decreases. A shadowing effect is observed for events arriving from a particular azimuth direction due to the presence of the electronics cylinder of the ANTARES line close to the PPMDOM. The final KM3NeT detection unit does not cause any kind of shadowing. In this case, the shadowing effect is taken into account in the simulation as a decrease of the PMT efficiency. There is a good agreement between data and the atmospheric muon simulation.

\section{Prototype Detection Unit}

The prototype detection unit was deployed during the night between May 6 and 72014 at the site located $80 \mathrm{~km}$ offshore Capo Passero, Sicily (latitude $36^{\circ} 17^{\prime} 50^{\prime \prime} \mathrm{N}$, longitude $15^{\circ} 58^{\prime} 45^{\prime \prime} \mathrm{E}$ ), at $3457 \mathrm{~m}$ depth. It is composed by 3 DOMs spaced by $36 \mathrm{~m}$ and kept vertical by two empty glass spheres that provide buoyancy, see Fig. 1b. The PPM-DU is a $160 \mathrm{~m}$ long; the full DU with 18 DOM will be $700 \mathrm{~m}$ long. The PPM-DU base is connected with the onshore station through 
an electro-optical cable of about $100 \mathrm{~km}$ length. DOM1 and DOM2 are equipped with ETEL D783FLA PMTs [6], while DOM3 contains Hamamatsu R12199-02 PMTs [10]. There are small differences in the global efficiency of the two kinds of PMTs that result in a slightly higher counting rate for Hamamatsu PMTs. Each DOM also contains:

- a LED nanobeacon for the inter-DOM time calibrations between different DOMs;

- a piezo for acoustic measurements;

- a compass and tiltmeter for relative positioning;

- a temperature and relative humidity sensor to monitor the conditions of the electronics inside the DOMs.

The PPM-DU is installed on the sea bed using a mechanical spherical structure that houses the full line. Once on the sea bottom, an acoustic release allows the unfurling of the line, and the mechanical spherical structure can be recovered at the sea surface. With the help of a Remotely Operated Vehicle (ROV), the electro-optical cable is then connected with the base of the PPM-DU, providing power supply and allowing communication with the onshore station. The geometry of the PMTs inside the three DOMs is the same of the PPM-DOM, and the DAQ electronics offshore and onshore are identical to the ones used in the DOM prototype. The FPGAs inside the DOMs arrange the signals from the 31 PMTs in timeslices of $\sim 134 \mathrm{~ms}$ and send them to shore. A dedicated software has been developed to synchronize offline the data stream of the three DOMs. In this prototype, the events are filtered from the uncorrelated background using a dedicated software and stored on disk. A simple L1 trigger is defined as two hits in the same DOM with a time difference smaller than $25 \mathrm{~ns}$. An L2 trigger stores physics event grouping all L1 triggers with a time difference smaller than $330 \mathrm{~ns}$ forward in time; this value is consistent with muons passing through the detector.

In order to double check the procedure of data taking and allow further studies, a reduced sample of untriggered data is saved every day. These untriggered data have been used to study the long term variations of the optical background, monitoring the single rates of the PMTs. For this study, the data stream has been redistributed in time widows of $100 \mathrm{~ms}$, and then compared with the standard timeslices of $134 \mathrm{~ms}$ to exclude any systematic effect. The average single rate distribution per time slices of $100 \mathrm{~ms}$ for one PMT of DOM 1 is shown in Fig. 3a. There are two evident contributions to the rate: one is a Gaussian distribution with the peak at $5.9 \mathrm{kHz}$ and the second is a high frequency tail. The Gaussian peak is due to the ${ }^{40} \mathrm{~K}$ decays, while the tail is related with transient bioluminescent phenomena.

The intra-DOM time calibration of the PMTs inside every DOM is done searching for ${ }^{40} \mathrm{~K}$ coincidences, in a similar way respect to the PPM-DOM. To calculate the inter-DOM time offsets (between DOMs) dedicated run with the LED nanobeacon activated are used. The LED nanobeacon installed in each DOM has a wavelength of $470 \mathrm{~nm}$ and is positioned in the top half of the DOM pointing upwards. The LED nanobeacons are controlled with adjustable frequency and intensity in order to illuminate the next DOM of the line without saturating the PMTs. The inter-DOM time offsets depend on the electronics plus the cable lengths. The travel time of light in sea water must be taken into account in this calibration procedure. For the travel time of the nanobeacon light, the 


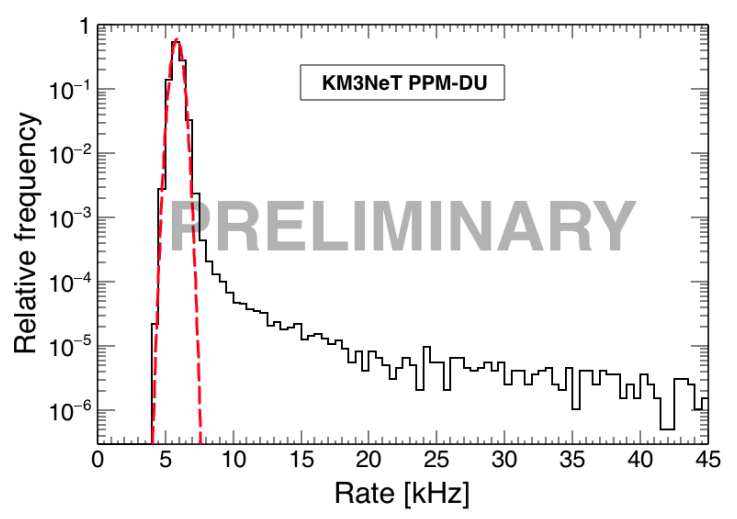

(a)

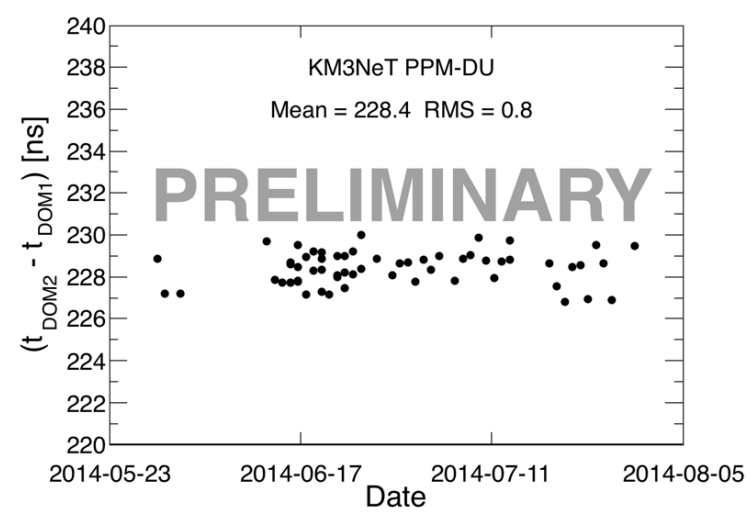

(b)

Figure 3: (a) PDF of the mean photon rate measured by PMT16 of DOM1. The rate is evaluated in time windows $100 \mathrm{~ms}$ large; the integral of the distribution is normalized to 1 . The gaussian fit (red dashed line) is superimposed and represent the optical background due to ${ }^{40} \mathrm{~K}$ decay. The tail on the right is due to transient bioluminescent phenomena of optical background. (b) Mean time offsets for DOM 2 with respect to DOM1 evaluated with the usage of the nanobeacon of DOM1 for different runs.

distance between the nanobeacon and the hit PMT is used. A fixed detector position is assumed, as the real time positioning system is not available for this prototype. In the calculation, the speed of $470 \mathrm{~nm}$ light (nanobeacon wavelength) in the water is used. The resulting mean time offsets for different runs are shown in Fig. 3b. The time accuracy achieved is of the order of $\sim 1$ ns.

A dedicated Monte Carlo simulates the expected atmospheric muon flux at a depth of $3457 \mathrm{~m}$, together with the optical background due to the ${ }^{40} \mathrm{~K}$ decay. The PMT characteristics and the optical water properties measured at the Capo Passero site [11] are taken into account in the simulation.

The optical background from ${ }^{40} \mathrm{~K}$ decays and bioluminescence dominate the coincidence rates in a DOM up to 5 coincidences, while muons are the dominant source at higher coincidences. Fig. 4a shows the rate of events as a function of the coincidence level in a DOMs for data and Monte Carlo simulation. The full Monte Carlo histograms reported in Fig. 4a refer to the sum of atmospheric muon events and ${ }^{40} \mathrm{~K}$ only events. No normalization factor is applied to the Monte Carlo events thus showing an absolute excellent agreement between data an Monte Carlo simulations.

Analogously to the PPM-DOM, a distribution of hits as a function of the PMT orientation is show in Fig. $4 \mathrm{~b}$ compared to the distributions of the muon Monte Carlo simulation. A cut for coincidence level $>7$ is applied. The resulting sample is essentially background free. The horizontal axis corresponds to the PMT location in each DOM: the first PMT is the one looking downward followed by groups of 6 PMTs each with different orientations. The directional sensitivity of the DOMs is clearly seen in the plot, reflecting the fact that atmospheric muons come from above. No shadowing effect is present, as expected. Muon simulations are in a good agreement with data.

An algorithm to reconstruct the zenith angle of the direction of atmospheric muons has been developed. This algorithm uses the information about the position of the three DOMs and the 


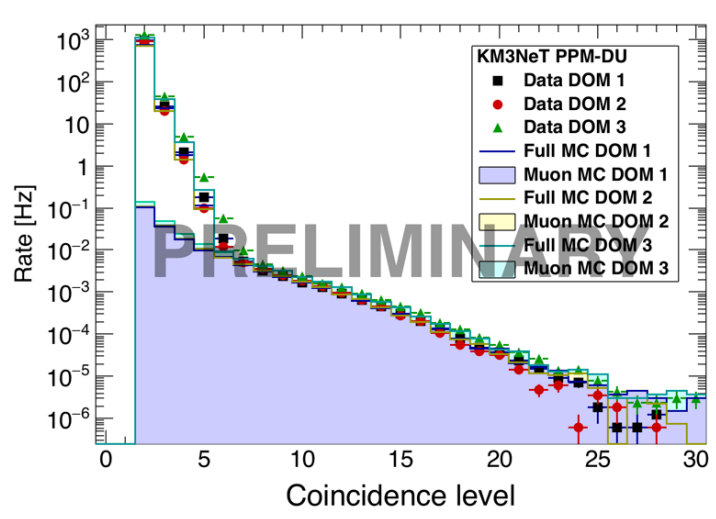

(a)

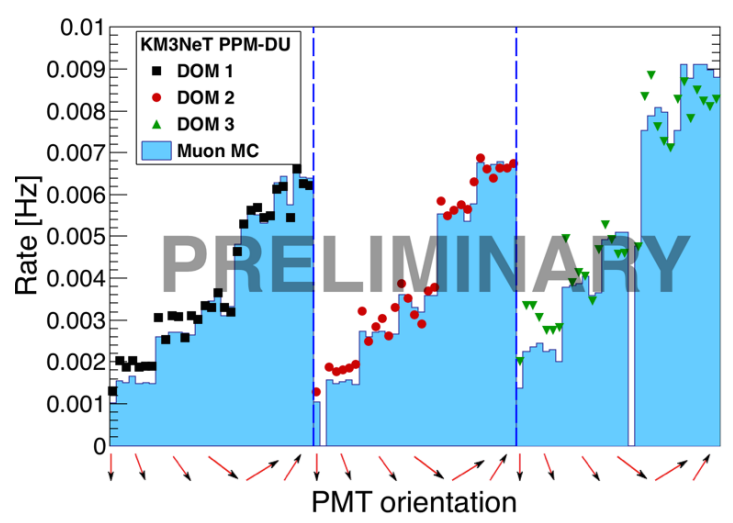

(b)

Figure 4: (a) Rate of events as a function of the number of PMTs in coincidence in a time window of $25 \mathrm{~ns}$, for the 3 DOMs of the PPM-DU compared to the Monte Carlo simulations. Points refer to data, histograms to Monte Carlo simulations. (b) Rate of hits on each DOM as a function of the PMT position for events with more than 7 coincidences on the respective DOM, compared with the muon simulations. No normalization factor is applied to Monte Carlo events.

time of the local coincidences detected. Hence, only muon events that trigger the three DOMs are reconstructed. The muon track can be parametrized by an offset in time $\left(t_{0}\right)$, its zenith angle $(\theta)$, the closest distance to the PPM-DU $\left(d_{0}\right)$, and the coordinate on the vertical axis $\mathrm{z}$ of the closest distance to the PPM-DU $\left(z_{0}\right)$. The signal expected time in a DOM is given by:

$$
t=\frac{\left(z-z_{0}\right) \cos \theta+\sqrt{n^{2}-1} \cdot \sqrt{d_{0}^{2}+\left(z-z_{0}\right)^{2} \sin ^{2} \theta}}{c}+t_{0}
$$

where $\mathrm{z}$ is the projection of the particle on the vertical axis, $n$ is the refractive index of sea water and $c$ is the speed of light. Only the the time differences between the two DOM pairs are used $\left(\Delta T_{12}\right.$ and $\left.\Delta T_{23}\right)$ and this can cause a degeneracy in the solution from the algorithm. Events with $-50 \mathrm{~ns}<\Delta T_{12}<155 \mathrm{~ns},-50 \mathrm{~ns}<\Delta T_{23}<165 \mathrm{~ns},\left(\Delta T_{23}-\Delta T_{12}\right)<10 \mathrm{~ns}$, and those with $d_{0}<10 \mathrm{~m}$ are kept, in order to exclude events with unphysical time differences and reduce the degeneracy. This selection keeps $\sim 2 / 3$ of all events with coincident signals in all 3 DOMs.

The times of the coincidences in the DOMs is compared to the expected signal times from a muon track. A $\chi^{2}$ minimization scan is performed in flat $\cos \theta$ between 0.5 and 1 (downgoing muons). The other 3 free parameters $\left(d_{0}, t_{0}, z_{0}\right)$ are varied keeping fixed the value of $\cos \theta$. The set of values resulting in the lowest $\chi^{2}$ are finally chosen as the track parameters. In Fig. 5a the difference between the reconstructed and the true zenith angle is plotted using Monte Carlo muon events; a FWHM of 7.6 degrees is achieved. A data sample with an equivalent livetime of $\sim 600$ hours has been used in comparison with the muon Monte Carlo simulation to test the reconstruction algorithm. The distribution of $\cos \theta$ for the selected events is shown for data and Monte Carlo in Fig. $5 \mathrm{~b}$ demonstrating an excellent agreement. 


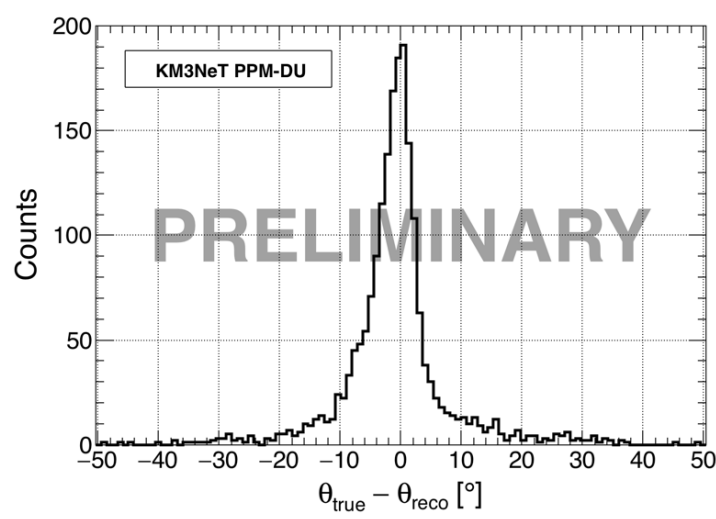

(a)

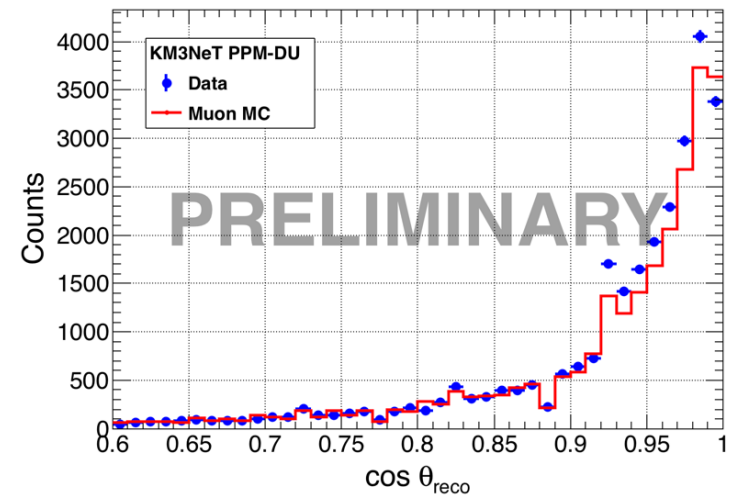

(b)

Figure 5: (a) Zenith angular resolution of the tracking algorithm obtained with Monte Carlo simulations. (b) Reconstructed $\cos \theta$ for data and Monte Carlo. A data sample with an equivalent livetime of $\sim 600$ hours is used in this comparison.

\section{Conclusions}

The experience gained with the prototypes presented in these proceedings paved the way to the forthcoming construction and installation of the full detection units in the KM3NeT marine sites in France and in Italy [12]. The prototype digital optical module demonstrates the capability to select a background free muon sample searching for local coincidences. The prototype detection units showed that a time calibration of the order of $1 \mathrm{~ns}$ is possible with the usage of local ${ }^{40} \mathrm{~K}$ coincidences and using the LED nanobecons for the inter-DOM time offsets; a tracking algorithm has been developed in order to reconstruct the zenith angle of atmospheric muons; dedicated Monte Carlo simulations showed a good agreement with the data.

\section{References}

[1] http://www.km3net.org

[2] S. Biagi, T. Chiarusi, P. Piattelli, and D. Real (KM3NeT Collaboration), The data acquisition system of the KM3NeT detector, in proceedings of $34^{\text {th }}$ ICRC, The Hague, The Netherlands, POS ( ICRC2015) 1172 (2015).

[3] S. Biagi and A. Orzelli (KM3NeT Collaboration), The Central Logic Board and its auxiliary boards for the optical module of the KM3NeT detector, JINST 9 (2014) C12033.

[4] S. Adrián-Martínez et al. (KM3NeT Collaboration), Deep sea tests of a prototype of the KM3NeT digital optical module, Eur. Phys. J. C 74 (2014) 3056.

[5] M. Ageron et al. (ANTARES Collaboration), ANTARES: the first undersea neutrino telescope, Nucl. Instr. Meth. A 656 (2011) 11.

[6] R. Bormuth et al. (KM3NeT Collaboration), Characterization of the ETEL and HZC 3-inch PMTs for the KM3NeT project, AIP Conference Proceedings 1630 (2014) 114.

[7] S. Adrián-Martínez et al. (KM3NeT Collaboration), Expansion cone for the 3-inch PMTs of the KM3NeT Optical Modules, JINST 8 (2013) T03006. 
[8] Y. Becherini, A. Margiotta, M. Sioli, and M. Spurio, A parameterisation of single and multiple muons in the deep water or ice, Astropart. Phys. 25 (2006) 1.

[9] B. Herold (KM3NeT Collaboration), Study of 40K-induced rates for a KM3NeT design option with multi-PMT optical modules, Nucl. Instr. Meth. A 626-627 (2011) S234.

[10] S. Aiello et al. (KM3NeT Collaboration), Characterization of the 80-mm diameter Hamamatsu PMTs for the KM3NeT project, AIP Conf. Proc. 1630 (2014) 118.

[11] The KM3NeT Collaboration, KM3NeT Technical Design Report, http://www. km3net.org/TDR/TDRKM3NeT.pdf.

[12] A. Creusot (KM3NeT Collaboration), Calibration, performances and tests of the first detection unit of the KM3NeT neutrino telescope, in proceedings of $34^{\text {th }}$ ICRC, The Hague, The Netherlands, PoS ( ICRC2015) 1154 (2015). 